\title{
Nonadherence to follow-up recommendations is common for dogs and cats undergoing orthopedic surgery
}

Kevin A. de Moya, DVM; Jade M. Reppenhagen, BS; Stanley E. Kim, BVSc, MS

Department of Small Animal Clinical Sciences, College of Veterinary Medicine, University of Florida, Gainesville, FL

Corresponding author: Dr. Kim (stankim@ufl.edu)

\section{OBJECTIVE}

To evaluate owner adherence to recommendations for follow-up examination of dogs and cats following orthopedic procedures and identify factors associated with adherence versus nonadherence.

\section{SAMPLE}

Medical records of 485 dogs and cats that underwent orthopedic surgery.

\section{PROCEDURES}

Cases were categorized as urgent or elective. Information obtained from the medical records consisted of species, age, body weight, proximity to the hospital, procedure cost, recommendations for coaptation, use of financial aid, and number of owners. Cases were considered adherent to follow-up recommendations if, at the latest visit or communication, no further visits were recommended. Cases were considered nonadherent if owners did not return for recommended follow-up visits.

\section{RESULTS}

Overall adherence to follow-up recommendations was $65.8 \%(319 / 485)$. Elective cases were I.6 times as likely to be adherent to follow-up recommendations as were urgent cases, dog cases were 2.4 times as likely to be adherent as were cat cases, and cases with multiple owners listed were 2.I times as likely to be adherent as were cases with I owner listed. Distance from the hospital had a statistically significant association with adherence, but the effect was not clinically important. Age, weight, coaptation, procedure cost, and use of financial aid were not significantly associated with adherence.

\section{CONCLUSIONS AND CLINICAL RELEVANCE}

The percentage of dogs and cats lost to follow-up following orthopedic surgery at an academic veterinary teaching hospital was substantial (166/485 [34.2\%]). Efforts to improve follow-up adherence are especially indicated for animals undergoing urgent procedures, animals with single owners, and cats.

$\mathbf{P}$ ostoperative instructions for human and veterinary patients that have undergone orthopedic surgery typically include a recommendation to return for follow-up examinations to monitor recovery and allow for early recognition and treatment of complications. Studies ${ }^{1,2}$ of human patients have demonstrated an association between lack of follow-up and poorer clinical outcomes among various patient populations, but to our knowledge, the existence of similar associations for veterinary patients has not yet been investigated. Previous investigators have identified several factors associated with adherence to recommendations for follow-up examinations in human medicine. ${ }^{3-6}$ For example, a retrospective study ${ }^{6}$ of human orthopedic patients identified tobacco use, living $>100$ miles from a medical clinic, and a lack of private insurance as factors associated with follow-up nonadherence. Clinician communication styles and institutional programs can also influence adherence to recommendations, including recommendations for follow-up examination. ${ }^{7,8}$
Adherence to recommendations in veterinary medicine has not been thoroughly described. In a study 9 of dogs that underwent surgery for apocrine gland anal sac adenocarcinoma, overall adherence to staging recommendations was $21 \%$ and adherence to treatment recommendations was $34 \%$. A study ${ }^{8}$ of general practitioners revealed that $30 \%$ of clients adhered to recommendations for dental or surgical treatments for their animals, but adherence was heavily influenced by the way in which the recommendations were communicated by the veterinarian. That study ${ }^{8}$ specifically investigated adherence to presurgical recommendations, and to the authors' knowledge, adherence to recommendations for postoperative recheck examinations in companion animals has not been well investigated. However, complete follow-up data seem difficult to obtain for veterinary orthopedic studies, ${ }^{10,11}$ and anecdotally, we have observed a concerningly low proportion of orthopedic cases treated at our hospital with a known final outcome. 
The objectives of the study reported here were to determine owner adherence rates to recommendations for follow-up examination of dogs and cats following orthopedic procedures and identify factors associated with adherence versus nonadherence. We hypothesized that cases involving urgent orthopedic procedures would have poorer follow-up adherence, compared with cases involving elective procedures. We also hypothesized that the distance required to travel to the veterinary hospital, initial procedure cost, animal body weight, species, and number of owners would be associated with follow-up adherence versus nonadherence.

\section{Materials and Methods}

\section{Case selection and medical records review}

Medical records of the University of Florida Small Animal Hospital were searched to identify dogs and cats that had undergone orthopedic surgery between February 2017 and February 2019. All procedures had been performed by a board-certified veterinary surgeon or a resident under the supervision of a board-certified veterinary surgeon. Recommended follow-up times were provided to owners at the time of discharge; however, owners were not obligated to make follow-up appointments. Typically, a veterinary student and house officer (intern or resident) were both responsible for communicating recommendations at the time of hospital discharge. A reminder telephone call by a veterinary technician was made to owners 3 business days prior to any scheduled visits. Owners who were due to return their animal for a follow-up examination but did not make an appointment were typically not contacted.

Cases included in the study were categorized as urgent or elective on the basis of the specific condition treated. Urgent cases included animals with acute ( $<2$ weeks old) fractures, luxations, and traumatic ligament and tendon injuries. Elective cases included animals treated for elbow dysplasia, hip dysplasia, cranial cruciate ligament rupture, patellar luxation, osteochondritis dissecans, angular limb deformity, and chronic ( $>2$ weeks old) fractures, luxations, and traumatic ligament and tendon injuries. Additional data collected from medical records consisted of animal age, species, and body weight; number of listed owners; distance between the owners' listed home address and the hospital; initial procedure cost; use of financial assistance (eg, use of the hospital compassion fund or acquisition of a third-party loan); whether mid-term ( $>2$ weeks) coaptation or bandaging was recommended; follow-up visit recommendations (weeks); and actual follow-up visit times.

Cases were considered adherent to follow-up recommendations if, at the latest visit or communication (by telephone or email), no further visits were recommended. Cases were considered nonadherent to follow-up recommendations if owners did not return with their animal for recommended follow-up visits to the hospital. Cases that were managed postoperatively by another clinic were deemed adherent to follow-up recommendations if medical records specifically indicated that ongoing care would be provided by another veterinary clinic. Cases were also deemed adherent to follow-up recommendations if major treatment recommendations such as revision surgery were declined. That is, a case could have an unsatisfactory outcome but still be deemed adherent to follow-up recommendations. For animals that underwent 2 separate, unrelated procedures $>2$ weeks apart, each procedure was considered to be a case, whereas for animals that underwent 2 procedures during the same hospital stay or $<2$ weeks apart, all procedures were considered a single case and the procedure that was deemed the most important was defined as the case procedure.

\section{Statistical analysis}

Continuous data are summarized as mean $\pm \mathrm{SD}$, and percentages of cases classified as adherent versus nonadherent were calculated. Logistic regression was used to assess factors potentially associated with nonadherence to follow-up recommendations. Specific risk factors assessed consisted of procedure type (elective vs urgent), species, age, weight, distance from the hospital, use of coaptation for $\geq 2$ weeks, initial procedure cost, use of financial assistance, and number of listed owners. Values of $P<0.05$ were considered significant. All statistical analyses were performed with standard software (Prism version 8.0; GraphPad).

\section{Results}

A total of 485 cases were included in the study. There were 314 (64.7\%) elective cases and 171 (35.3\%) urgent cases representing 451 (93.0\%) dogs and $34(7.0 \%)$ cats. The most common elective procedure was tibial plateau leveling osteotomy $(\mathrm{n}=$ 167), and the most common urgent procedure was long bone fracture repair (142). Other elective procedures included medial patellar luxation correction $(n=39)$, femoral head and neck ostectomy (27), total hip arthroplasty (20), corrective osteotomy for angular limb deformity (13), shoulder arthroscopy (11), tarsal arthrodesis (10), lateral suture stabilization (10), elbow arthroscopy (7), carpal arthrodesis (6), and center of rotation and angulation-based leveling osteotomy (4). Other urgent procedures included mandibular fracture repair $(n=16)$ and pelvic fracture repair (13). Mean $\pm \mathrm{SD}$ age at the time of surgery was $4.4 \pm 3.6$ years (range, 0.3 to 15 years). Mean body weight was $22 \pm 16 \mathrm{~kg}$ (range, 1 to $79 \mathrm{~kg}$ ). Mean distance from the hospital was $108.6 \pm 137.6$ miles (range, 3 to 1,316 miles). Mean initial procedure cost was $\$ 3,455 \pm 1,558$ (range, $\$ 1,402$ to $\$ 22,227$ ). Coaptation for $\geq 2$ weeks was recommended in 40 (8.2\%) cases. Financial assistance was used in 120 (24.7\%) 
Table I-Results of multivariable logistic regression modeling to identify risk factors associated with adherence to recommendations for follow-up visits for $\mathbf{4 8 5}$ dogs and cats that underwent orthopedic procedures at an academic veterinary teaching hospital.

\begin{tabular}{|c|c|c|}
\hline Variable & OR (95\% CI) & $P$ value \\
\hline $\begin{array}{l}\text { Procedure type (elective } \\
\text { vs urgent) }\end{array}$ & 1.626 (I.027-2.578) & 0.04 \\
\hline Species (dog vs cat) & $2.423(\mathrm{I} .1 \mathrm{I}-5.3 \mathrm{II})$ & 0.03 \\
\hline Age $(y)$ & $0.9919(0.9386-1.049)$ & 0.76 \\
\hline Weight (kg) & $0.9894(0.9753-1.004)$ & 0.14 \\
\hline $\begin{array}{l}\text { Distance from hospital } \\
\text { (miles) }\end{array}$ & $0.9984(0.9968-0.9998)$ & 0.04 \\
\hline Coaptation for $\geq 2$ wk & I.38I (0.6582-3.094) & 0.41 \\
\hline Initial procedure cost & $0.9999(0.9998-1.0000)$ & 0.38 \\
\hline Use of financial assistance & $0.851(0.5425-1.344)$ & 0.49 \\
\hline Number of listed owners & $2.096(1.413-3.127)$ & $<0.01$ \\
\hline
\end{tabular}

cases. A single owner was listed for 254 (52.4\%) cases, and multiple owners were listed for 231 (47.6\%).

Overall adherence to follow-up recommendations was $65.8 \%(319 / 485)$. Of the 166 cases nonadherent to follow-up recommendations, 83 (50\%), $60(36 \%), 8(5 \%)$, and 7 (4\%) missed their first, second, third, and fourth or later follow-up visit, respectively. A higher proportion of elective cases adhered to follow-up recommendations (219/314 [69.7\%]), compared with urgent cases (100/171 [58.5\%]). Elective cases were 1.6 times as likely to be adherent to follow-up recommendations as were urgent cases (Table I), dog cases were 2.4 times as likely to be adherent as cat cases, and cases with multiple owners listed were 2.1 times as likely to be adherent as cases with only 1 owner listed. Distance from the hospital had a statistically significant association with adherence to follow-up recommendations; however, the effect size was small ( $0.1 \%$ change in odds per 1 -mile increase in distance). Age, weight, use of coaptation for $\geq 2$ weeks, initial procedure cost, and use of financial assistance were not significantly associated with adherence to follow-up recommendations.

\section{Discussion}

In the present study, the percentage of dogs and cats lost to follow-up following orthopedic surgery at an academic veterinary teaching hospital was substantial (166/485 [34.2\%]), and adherence to followup recommendations was particularly poor for urgent cases (71/171 [41.5\%]). Further, a large percentage of cases designated as nonadherent to follow-up recommendations (83/166 [50\%]) did not return for $>1$ recommended follow-up visit. We also found that cases with a single owner and cat cases specifically were more likely to be nonadherent. Finally, although there was a statistically significant association between nonadherence and greater distance between listed home address and the hospital, the effect size was clinically insignificant.
In the present study, follow-up adherence was defined simply by whether an animal was returned for a follow-up appointment. We used this criterion because we were mainly interested in the percentage of treated cases with a known outcome. The low rates of follow-up adherence were concerning but not surprising. For instance, it is common for retrospective studies in veterinary orthopedics to exclude large numbers of cases owing to inadequate followup. ${ }^{10}$ Our rates of nonadherence to follow-up recommendations appeared comparable to those reported for human patients that have undergone orthopedic trauma surgery, for whom follow-up adherence at the first scheduled postoperative recheck appointment has been reported to be $66.9 \% .^{6}$

Cases undergoing elective procedures had better follow-up adherence than did cases undergoing urgent procedures in the present study. This was an expected finding and agreed with our hypothesis. It is likely that owners of animals in the urgent and elective groups represented 2 different owner populations. Owners of elective cases may be more financially able to comply with follow-up recommendations, whereas owners of urgent cases may be more representative of a broader owner population. Because elective procedures by definition are not essential, owners have time to research and prepare for the procedure prior to proceeding. The higher follow-up rates for cases ungergoing elective procedures may therefore be a reflection of owners being properly educated and financially prepared for the procedure. For cases considered urgent, owners may have had less time to consider long-term care and associated follow-up costs for the procedure recommended. Urgent cases were associated with trauma, and the nature of those injuries and procedures may have resulted in greater variability in follow-up visits relative to recommendations for elective cases. In addition, clinician-related factors may have played an important role. The manner in which recommendations are made by clinicians can have a profound effect on owner or patient adherence, ${ }^{8}$ and it is possible that communication with clients was not as well developed for urgent cases as for elective cases.

In the present study, dog cases were more than twice as likely to have been adherent to followup recommendations as were cats. However, cats accounted for only $7 \%$ of the study population, which reflected the overall caseload of our referral small animal hospital. Consequently, fear-free environmental adaptations for cats are not featured prominently in our hospital. Stress-related considerations are a wellknown deterrent for cats requiring veterinary visits, and practices such as pheromonotherapy and felinespecific examination rooms can reduce stress scores in cats. ${ }^{12}$ Whether providing such adaptations and educating owners about their benefits could impact follow-up adherence in cats requires further investigation. In the recent AVMA Pet Ownership and Demograpbic Sourcebook, ${ }^{13}$ cats were significantly 
more likely to be considered a companion rather than a family member, compared with dogs, and lower follow-up adherence rates may be reflective of the difference in the animal-owner bond for dogs versus cats.

Cases that had $\geq 2$ owners listed were twice as likely in the present study to be adherent to followup recommendations as were cases with only a single owner. Reasons for this finding are unclear, but it could have reflected the benefits of having multiple individuals responsible for the care of an animal. For instance, multiple owners may have greater flexibility in their schedules and, thus, be better able to comply with follow-up visits. Additionally, 2 owners may typically have more income, compared with a single owner and may therefore be more financially able to comply with follow-up recommendations and their associated costs. It is important to note that actual owner financial status was unknown in the present study, and no other patterns associated with finances (such as initial cost of the procedure and use of financial assistance) were observed. Furthermore, the number of listed owners may not accurately reflect the number of actual owners, as there are no established rules for capturing all legal owners in our medical record system.

Although distance from hospital had a statistically significant effect on adherence to follow-up recommendations in our study, the effect size was not clinically significant. We therefore rejected the hypothesis that longer travel distances lead to poorer adherence to follow-up recommendations. A study ${ }^{14}$ investigating barriers to follow-up after general surgery procedures in people found that increased travel time was associated with a reduced likelihood of adhering to postoperative follow-up recommendations. The lack of association between travel distance and follow-up adherence in our study could be explained by the fact that owners were already willing to travel for the index treatment at our hospital over other more local specialty clinics, and owners who were willing to travel to our facility were not any more averse to returning for follow-up visits. This explanation may also address the lack of association between initial procedure cost and follow-up adherence, in that owners who were willing to pay for more expensive procedures were just as willing to pay for follow-up visits.

Our study did not provide any insight into the benefits of follow-up visits. Follow-up visits were recommended for the purposes of optimizing clinical outcomes by monitoring progress and adjusting therapeutic interventions; however, it is not possible to determine from this study whether nonadherent cases had poorer outcomes than adherent cases. This observation highlights one of the main motivations for conducting the present study: that the effects of our interventions remain unknown without proper follow-up.

The retrospective nature of the present study was a major limitation. Some animals considered lost to follow-up may have still obtained proper postoperative and ongoing care at other veterinary clinics without keeping our hospital informed. The initial procedure cost may not have been reflective of the original financial estimate provided, and the initial estimate (rather than final cost) may have had a stronger influence on whether a client was able to afford follow-up visits. We were not able to investigate the effect of pet insurance, because this information was not available in our medical records system. Unsurprisingly, previous studies ${ }^{6,15}$ in human medicine have demonstrated a poorer adherence to follow-up recommendations among individuals who do not have health insurance, and we suspect a similar effect would be apparent for veterinary patients. Importantly, adherence is influenced by both clinician- and client-related factors, ${ }^{8}$ and our study design precluded the ability to investigate important clinician-related factors such as the ability to communicate the value of follow-up visits. All cases were treated at a single institution, and our results may not be representative of other types of hospitals. For example, hospitals with stronger ties to referring veterinarians may have fewer cases nonadherent to follow-up recommendations. This may be especially relevant when bandage changes, which are often performed by a local veterinarian, are recommended. Our population was heterogenous, including cases that underwent a wide array of orthopedic procedures, and the sample size was too small to parse out differences in follow-up rates according to anatomic site or surgical procedure.

In conclusion, results of the current study indicated that a large percentage of dogs and cats will be lost to follow-up after orthopedic surgery, and we were able to identify risk factors associated with nonadherence to follow-up recommendations. Future studies should investigate adherence in other veterinary hospitals, such as private practices, and strategies to improve follow-up adherence.

\section{Acknowledgments}

No compensation or funding was received in connection with this study. The authors declare that there were no conflicts of interest.

\section{References}

1. Keren D, Matter I, Rainis T, et al. Getting the most from the sleeve: the importance of post-operative follow-up. Obes Surg. 2011;21:1887-1893.

2. Karmisholt J, Andersen S, Laurberg P. Variation in thyroid function in subclinical hypothyroidism: importance of clinical follow-up and therapy. Eur J Endocrinol. 2011;164: 317-323.

3. Strauss K, MacLean C, Troy A, et al. Driving distance as a barrier to glycemic control in diabetes. J Gen Intern Med. 2006;21:378-380.

4. Gabayan GZ, Asch SM, Hsia RY, et al. Factors associated with short-term bounce-back admissions after emergency department discharge. Ann Emerg Med. 2013;62:136-144.e1.

5. Peipins LA, Graham S, Young R, et al. Time and distance barriers to mammography facilities in the Atlanta metropolitan area.J Community Health. 2011;36(4):675-683. 
6. Whiting PS, Greenberg SE, Thakore RV, et al. What factors influence follow-up in orthopedic trauma surgery? Arch Orthop Trauma Surg. 2015;135:321-327.

7. Hendrickson SB, Simske NM, DaSilva KA, Vallier HA. Improvement in outpatient follow-up with a postdischarge phone call intervention. J Am Acad Ortbop Surg. 2020;28:e815-e822.

8. Kanji N, Coe JB, Adams CL, Shaw JR. Effect of veterinarianclient-patient interactions on client adherence to dentistry and surgery recommendations in companion-animal practice. J Am Vet Med Assoc. 2012;240:427-436.

9. Chambers AR, Skinner OT, Mickelson MA, et al. Adherence to follow-up recommendations for dogs with apocrine gland anal sac adenocarcinoma: A multicentre retrospective study. Vet Comp Oncol. 2020;18(4):683-688.

10. Watrous GK, Moens NM. Cuttable plate fixation for small breed dogs with radius and ulna fractures: Retrospective study of 31 dogs. Can Vet J. 2017;58(4):377-382.
11. Forster KE, Wills A, Torrington AM, et al. Complications and owner assessment of canine total hip replacement: a multicenter internet based survey. Vet Surg. 2012;41:545-550.

12. Lloyd JK. Minimizing stress for patients in the veterinary hospital: why it is important and what can be done about it. Vet Sci. 2017;4(2):1-22.

13. American Veterinary Medical Association. AVMA Pet Ownership and Demographics Source Book. American Veterinary Medical Association. 2018; 169.

14. Harkey K, Kaiser N, Inman M, et al. "Are we there yet?"factors affecting postoperative follow-up after general surgery procedures. Am J Surg. 2018;216(6):1046-1051.

15. Stone ME Jr, Marsh J, Cucuzzo J, Reddy SH, Teperman S, Kaban JM. Factors associated with trauma clinic followup compliance after discharge: experience at an urban Level I trauma center. J Trauma Acute Care Surg. 2014;76: 185-190. 